\title{
EDITORIAL
}

\section{Cardiac surgery and the brain: differences between adult and paediatric studies}

\section{C Bellinger}

Heart 2003;89:365-366

Evidence is growing that patients with congenital heart disease who undergo surgery may be at increased risk of neurodevelopmental dysfunctions, particular paediatric survivors. However, paediatric studies involve different challenges from those conducted on adults

n this issue, Dittrich and colleagues ${ }^{1}$ report a study examining neurodevelopmental outcomes at one year of age in children with congenital heart defects who underwent reparative or palliative surgery in infancy. It adds to the burgeoning literature suggesting that such patients are at increased risk of adverse sequelae. Similar studies have been reported on patients who underwent cardiac surgery as adults, but it is important to note that paediatric studies involve a different set of challenges, involving the study designs that can be implemented, the statistical analyses required, and the inferences that investigators draw.

Studies of adult patients often involve administration of the same neuropsychological battery before surgery and again, sometimes repeatedly, over a follow up interval of weeks to years. ${ }^{2} \mathrm{Hav}$ ing each patient thus serve as his or her own control pays dividends in terms of statistical power, insofar as use of change scores effectively removes the variability in test scores that reflects betweenpatient differences in factors that affect overall level of performance (for example, education, sex, age). Considerable attention has therefore been devoted to determining valid methods for identifying a significant change in performance (for example, the reliable change index, a decline of 1 or more standard deviations on $20 \%$ of the tests administered, a $20 \%$ change in $20 \%$ of tests administered, and so on). ${ }^{3-5}$

\section{CIRCUMSTANCES DIFFER IN PAEDIATRIC STUDIES}

Such changes in scores usually cannot be used when the patients are children. First, they are often acutely ill before surgery, sometimes sedated and ventilated, reducing the validity of functional assessments performed at that time. Second, some forms of critical congenital heart disease must be repaired hours to weeks after birth, and the functional testing that can be completed on even healthy full term neonates is severely limited in terms of scope and predictive validity for later outcomes. Complex late outcomes in which we are often most interested, such as intelligence and academic achievement, simply cannot be tested in infancy and valid early david.bellinger@

.................
Boston MA 02115, Boston MA 02115 , markers of such skills are not available. Moreover, cardiac surgery might cause damage that remains "silent" until the child is required to learn such complex skills. This leaves investigators in the difficult position of trying to identify neurodevelopmental deficits without having a baseline measure of performance (that is, pre-surgery). Multivariate statistical methods must be used to distinguish the portion of between-child variability in post-surgery scores that is attributable to aspects of the surgical intervention (for example, the duration of total circulatory arrest) from the between-child variability in post-surgery scores that is attributable to the myriad other influences on children's neurodevelopment (for example, social class, home environment). Conducting a study as a clinical trial, with random assignment to treatment groups, ${ }^{67}$ is helpful in this regard because, if all goes well, the distribution of non-surgical determinants of between-child variability will be comparable across treatment groups. It does not, however, solve the problem of the missing baseline.

Because of the need to give anticipatory guidance to parents and primary care providers, an important goal of many paediatric studies is to draw inferences about the range of neurodevelopmental function that can be expected after surgery. For an adult, level of function before surgery provides, under most circumstances, an upper boundary on predicted performance postsurgery, obviating the need for an external control group. In paediatric studies, the non-availability of pre-surgery assessments of the late outcomes of interest makes the assembly of such a control group important, however. As in all clinical research, choosing an appropriate control group is fraught with hazard. ${ }^{8}$ Dittrich and colleagues ${ }^{1}$ assembled a control group of 20 outpatients with minor or no cardiac disease. This strategy was well considered, although it is important to bear in mind that the lower scores observed among the surgical patients might not be attributable only to their having undergone surgery but, as well, to the fact that they had cardiac disease severe enough to require surgery (that is, confounding by indication).

Dittrich and colleagues ${ }^{1}$ also calculated the score representing 2 standard deviations (SD) below the control mean in order to determine the frequency of surgical patients with "developmental delay". A large percentage (27\%) were so identified, particularly among the palliative surgery subgroup (63\%). This is alarming, but the SD of the controls was only 6.6 (against a mean of 106.7), so scores below 94 were considered indicative of developmental delay. While the 
authors make a strong case for the internal validity of their comparison of patients to controls, the argument for its external validity, and its implied clinical implications, is less convincing. A child who presents to a neurodevelopmental clinic with a score of 93 is unlikely to be diagnosed as developmentally delayed. In the general population, the mean and SD on the Griffith developmental scales used in this study are generally about 100 and 15, respectively. Why the control group SD was so small is unclear. With a mean of 99 and SD of 10.6 among children in the surgery group, assuming normality, perhaps $1-2 \%$ scored less than 70 ( 2 SD). This percentage, derived on the basis of the SD of a large standardisation sample, is certainly less alarming than is $27 \%$. If patients' post-surgery scores could have been compared to their pre-surgery scores, as is possible with adults, whether or not the SD used is appropriate would not be an issue.

\section{BRAIN INSULT}

Finally, in adult cardiac surgery, any brain insult will affect an organ that is presumed to have previously been normal and completely developed. A "static neuropsychological deficit" model $^{9}$ is thus applicable. A brain insult that occurs during infant cardiac surgery affects an organ that will, under normal circumstances, continue to undergo enormous change well into the second decade. Thus, it might alter the way in which the developmental programme plays out post-surgery. (Of course the increased plasticity implied might be protective in some respects.) Moreover, the brain-behaviour relations underlying complex cognitive processes differ by age, ${ }^{10}{ }^{11}$ with neural substrates generally being more widely distributed in children than in adults. ${ }^{12}$ Thus a given insult would be expected to have different effects in children and adults, confirming that children are not simply "little adults".

Surgeons are now able to repair forms of congenital heart disease that, until recently, were associated with high rates of mortality. As Dittrich and colleagues ${ }^{1}$ demonstrate, survivors may be at increased risk of neurodevelopmental dysfunctions. Identifying ways in which to minimise the frequency and severity of such outcomes should become a high priority. All infants who undergo cardiac surgery should be under increased developmental surveillance.

\section{REFERENCES}

1 Dittrich H, Bührer C, Grimmer I, et al. Neurodevelopment at 1 year of age in infants with congenital heart disease. Heart 2003;89:436-41.

2 Newman MF, Kirchner JL, Phillips-Bute B, et al. Longitudinal assessment of neurocognitive functioning after coronary-artery bypass surgery. N Engl J Med 2001:344:395-402

3 Blumenthal JA, Mahanna EP, Madden DJ, et al. Methodological issues in the assessment of neuropsychologic function after cardiac surgery. Ann Thorac Surg 1995;59:1345-50.

4 Temkin NR, Heaton RK, Grant l, et al. Detecting significant change in neuropsychological test performance: a comparison of four models. J Int Neuropsychol Soc 1999;5:357-69.

5 Barr W. Neuropsychological testing for assessment of treatment effects: methodologic issues. CNS Spectrums 2002;7:300-6.

6 Newburger JW, Jonas RA, Wernovsky G, et al. A comparison of the perioperative neurologic effects of hypothermic circulatory arrest versus low-flow cardiopulmonary bypass in infant heart surgery. N Engl J Med 1993;329:1057-64.

7 Bellinger DC, Jonas RA, Rappaport LA, et al. Developmental and neurologic status of children after heart surgery with hypothermic circulatory arrest or low-flow cardiopulmonary bypass. N Engl J Med $1995 ; 334: 549-55$

8 Rothman KJ, Greenland S. Case-control studies. In: Rothman KJ, Greenland S. Modern epidemiology, 2nd ed. New York: Lippincott Williams \& Wilkins, 1998:93-114.

9 Oliver A, Johnson MH, Karmiloff-Smith A, et al. Deviations in the emergence of representations: a neurocontructivist framework for analyzing developmental disorders. Developmental Science 2000;3: 1-40.

10 Gaillard WD, Hertz-Pannier L, Mott SH, et al. Functional anatomy of cognitive development. FMRI of verbal fluency in children and adults. Neurology 2000;54:180-5

11 Schlagger BL, Brown TT, Lugar HM, et al. Functional neuroanatomical differences between adults and school-age children in the processing of simple words. Science 2002;296:1476-9.

12 Stiles J. Neural plasticity and cognitive development. Devel Neuropsychol 2000;18:237-72.

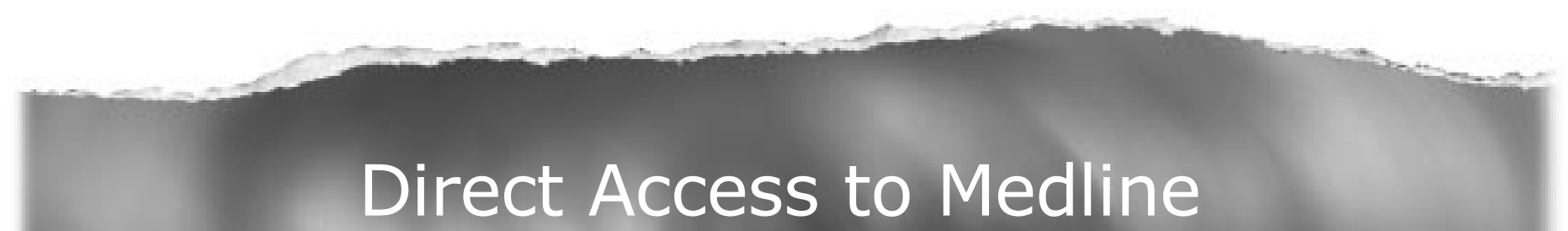

Medline

Link to Medline from the homepage and get straight into the National Library of Medicine's premier bibliographic database. Medline allows you to search across 9 million records of bibliographic citations and author abstracts from approximately 3,900 current biomedical journals.

\section{www.heartjnl.com}

